\title{
Toll-like receptor 2 in promoting angiogenesis after acute ischemic injury
}

\author{
YIFEI XU* , YING ZHOU* , HAIYAN LIN, HAIYANG HU, YUXING WANG and GENG XU \\ Department of Cardiology, The Second Affiliated Hospital, Zhejiang University \\ College of Medicine, Hangzhou, Zhejiang 310009, P.R. China
}

Received October 31, 2012; Accepted December 6, 2012

DOI: $10.3892 /$ ijmm.2013.1240

\begin{abstract}
Angiogenesis is an important mechanism that protects tissue against necrosis following acute ischemic injury. The aim of this study was to investigate whether the Toll-like receptor 2 (TLR2) signaling pathway is involved in angiogenesis following ischemic injury by cell migration and lymphocyte invasion assays in vitro, and a mouse model of hindlimb ischemia by ligation in vivo, respectively. To assess the potential role of TLR2 activation in endothelial cell permeability, HUVECs were pretreated with Pam3CSK4 and analyzed using wound repair and transwell assays. The results showed that the TLR2 agonist induced human umbilical vein endothelial cell (HUVEC) migration and increased the permeability of HUVECs to lymphocyte. The lymphocyte invasion of TLR2 knockout (TLR2 $2^{-/}$) mice was inhibited as compared to that of wild-type (WT) mice. In the mouse model of hindlimb ischemia by ligation, blood perfusion of operated limbs was significantly lower in TLR $2^{-/-}$compared to WT mice, 7 and 14 days after ligation. TLR $2^{-/-}$mice showed a decreased CD31 expression in ischemic gastrocnemius at 7 and 14 days after ligation, reduced interleukin-6 (IL-6) level and lowered tumor necrosis factor- $\alpha$ (TNF- $\alpha$ ) levels. These findings demonstrated that TLR2 activation promotes cell
\end{abstract}

Correspondence to: Professor Geng Xu, Department of Cardiology, The Second Affiliated Hospital, Zhejiang University College of Medicine, 88 Jiefang Road, Hangzhou, Zhejiang 310009, P.R. China E-mail: xugenghz@gmail.com

${ }^{*}$ Contributed equally

Abbreviations: TLR2, Toll-like receptor 2; HUVEC, human umbilical vein endothelial cell; TLR2 ${ }^{-/}$, TLR2 knockout; WT, wild-type; CD31, cluster of differentiation 31; IL-6, interleukin-6; TNF- $\alpha$, tumor necrosis factor- $\alpha$; NF- $\kappa \mathrm{B}$, nuclear factor- $\kappa \mathrm{B} ; \mathrm{AP}-1$, activator protein-1; $\mathrm{I} / \mathrm{R}$, ischemia/reperfusion; MAPK, mitogen-activated protein kinase; OCT, optimal cutting temperature; PBS, phosphate-buffered saline; ELISA, enzyme-linked immunosorbent assay; HP, high power lens; DAMPs, damage-associated molecular patterns

Key words: Toll-like receptor 2, angiogenesis, ischemia, lymphocyte, tumor necrosis factor- $\alpha$, interleukin- 6 migration, cell permeability and the lymphocyte invasion of endothelial cells. TLR2 activation promotes angiogenesis in vivo, which may be associated with the serum of TNF- $\alpha$ levels and IL-6 release.

\section{Introduction}

Atherosclerosis occurs in all arteries, inducing acute arterial occlusion, which may lead to a sudden decreased blood flow in tissue, resulting in cell necrosis and even death (1-3). The collateral circulation produced in the ischemic region may limit infarction size and improve organ function. However, the slow process of collateral circulation formation is not sufficient for the needs of ischemic tissue. Therefore, therapeutic angiogenesis is necessary (4). Toll-like receptor 2 (TLR2), whose coding gene is located on chromosome $4 q 32$ (5), is an important member of the TLR family. TLR2, expressed most abundantly in peripheral blood leukocytes (5), is able to recognize bacteria and endogenous ligands that activate nuclear factor- $\kappa \mathrm{B}(\mathrm{NF}-\kappa \mathrm{B})$ and activator protein-1 (AP-1) (6), playing an important role in inflammation, immunity response and tumorigenesis (7).

Following ischemic injury, apoptotic cells may induce a wound healing response, release of inflammatory cytokines and the recruiting of inflammatory cells, all of which are important processes in angiogenesis (8). TLR2 deficiency induced renal injury, in renal ischemia/reperfusion (I/R) $(9,10)$, and reduced infarction size after I/R (11). Administration of Pam3CSK4 prior to myocardial I/R reduced infarction size, improved cardiac function, and decreased leukocyte infiltration to ischemic tissues (12). Treatment of mice with Pam3CSK4 also induced protection against cerebral ischemic injury (13) and attenuated cardiac dysfunction in septic mice (14). Helicobacter pylori was reported to activate the mitogen-activated protein kinase (MAPK) cascade through TLR2, thereby contributing to cancer cell invasion and angiogenesis (15). The protective role of TLR2 against ischemic injury has been clarified, however, the contribution of TLR2 following the process of recovery from ischemia was found to be different in various organs (5). Therefore, the manner in which TLR2 impacts on ischemic tissues in vivo remains to be determined.

This study aimed to clarify the potential role of TLR2 in angiogenesis following ischemic injury. Human umbilical vein 
endothelial cells (HUVECs) were used to assess the role of TLR2 on cell migration, permeability and lymphocyte invasion in vitro. TLR2 knockout (TLR2 $2^{-/}$) mice and wild-type (WT) mice were used to clarify the role of TLR2 in neovascularization following ischemic injury in a mouse model of hindlimb ischemia by ligation.

\section{Materials and methods}

Cell culture. HUVECs were purchased from the Shanghai Touching Technology Co., Ltd. (Shanghai, China) and maintained in RPMI-1640 medium (Genom Biopharmaceutical Technology Co., Ltd., Hangzhou, China) supplemented with $10 \%$ fetal bovine serum (FBS) (Sijiqing Co., Ltd., Hangzhou, China). The cells were grown at $37^{\circ} \mathrm{C}$ in a humidified incubator in $5 \% \mathrm{CO}_{2}$ and $95 \%$ air.

Wound repair assays. HUVECs were seeded into 6-well plates and grown to confluence. A single scratch wound was made through the middle of each well with a sterile pipette tip. Cells were cultured with RPMI-1640 medium without FBS, and stimulated for $30 \mathrm{~h}$ with $1 \mu \mathrm{g} / \mathrm{ml} \mathrm{Pam3CSK} 4$ (Invivogen, San Diego, CA, USA) and $2 \mu \mathrm{g} / \mathrm{ml}$ Pam3CSK4, respectively. Migration of HUVECs across the wound margins from 18-30 $\mathrm{h}$ was assessed and photographed by inverted microscopy (CKX41; Olympus).

Transwell assay on HUVEC permeability and lymphocyte invasion. The Transwell chamber with $3 \mu \mathrm{m}$ membranes (Millipore, USA) was pre-coated with $0.2 \%$ gelatin (Sigma, St. Louis, MO, USA). Lymphocytes were separated from peripheral blood of mice using the density gradient centrifugation method. To assess the effect of Pam3CSK4 on the permeability of HUVECs to lymphocytes, HUVECs were seeded in the upper chamber at a density of $5 \times 10^{4} /$ well, and half of the cells were then treated with Pam3CSK4 $(1 \mu \mathrm{g} / \mathrm{ml})$ for $24 \mathrm{~h}$ at $37^{\circ} \mathrm{C}$. Lymphocytes, separated from WT mice, were placed in the upper chambers at a density of $5 \times 10^{4} /$ well with $100 \mu 12 \%$ FBS RPMI-1640 medium, while the lower chamber contained $600 \mu 1$ 10\% FBS RPMI-1640 medium. To evaluate the effect of Pam3CSK4 on lymphocyte invasion, HUVECs were seeded at a density of $1 \times 10^{5} /$ well in the upper chamber for $24 \mathrm{~h}$ at $37^{\circ} \mathrm{C}$. Lymphocytes, separated from the peripheral blood of WT and TLR $2^{-1-}$ mice, were placed in different upper chambers at a density of $5 \times 10^{5} /$ well with $100 \mu 12 \%$ FBS RPMI-1640 medium, while the lower chamber contained $600 \mu 1$ 10\% FBS RPMI-1640 medium and $20 \mathrm{ng} / \mathrm{ml}$ vascular endothelial growth factor. Migration was carried out for $24 \mathrm{~h}$ at $37^{\circ} \mathrm{C}$ and $5 \% \mathrm{CO}_{2}$. The medium was then removed. Transwell membranes of the upper chambers were fixed in $4 \%$ paraformaldehyde and stained with crystal violet. The HUVECs were removed and migrated cells on the membrane were quantified using an inverted microscope (CKX41; Olympus).

Animals. WT mice (C57BL/6, 18-25 g, 8-10 weeks old) were obtained from the Laboratory Animal Center at the Zhejiang Chinese Medical University and TLR2 ${ }^{-1-}$ mice

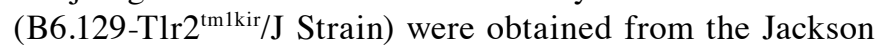
Laboratory. The two types of mice were maintained under specific pathogen-free conditions and received a standard diet and water ad libitum in the Laboratory Animal Center at the First Affiliated Hospital of the College of Medicine, Zhejiang University. Experimental studies were carried out in accordance with the Guide for the Care and Use of Laboratory Animals published by the National Institutes of Health (NIH publication no. 85-23).

Hindlimb ischemia by ligation (16) and blood flow monitoring. Under sterile conditions, proximal and distal portions of the left femoral artery were exposed and ligated, followed by the excision of the artery between ligation points. The superficial branch of the femoral artery was also ligated. A sham procedure (dissection of vessels without ligation) was performed on the right leg. Blood flow in the two limbs was measured using a Laser Doppler blood perfusion monitor (PeriFlux System 5000, Perimed AB) prior to ligation, and 1, 3,7 and 14 days after ligation (TLR2 ${ }^{-/}$vs. WT mice, respectively). Measurement was performed on six different locations on the leg, and the mean value of blood perfusion was used to evaluate the blood flow of each hindlimb. The gastrocnemius and serum were also harvested for analysis.

Immunohistochemistry. Ischemic gastrocnemiuses were collected 1, 3, 7 and 14 days after ligation, and fixed in 4\% paraformaldehyde. Optimal cutting temperature (OCT) sections $(5 \mu \mathrm{m})$ were sliced and placed on glass slides coated with polylysine. The streptavidin-biotin complex technique was used for immunohistochemistry assay. Endogenous peroxidase activity was blocked using $3 \% \mathrm{H}_{2} \mathrm{O}_{2}$. The sections were incubated with primary antibody against the mouse cluster of differentiation 31 (CD31) (Abcam, USA) at room temperature for $1 \mathrm{~h}$. Samples were then washed with phosphate-buffered saline (PBS) and incubated with secondary goat anti-rabbit antibody and streptavidin-biotin-peroxidase complexes. The samples were visualized using a microscope (DM 2500; Leica, Germany).

Enzyme-linked immunosorbent assay (ELISA). Mouse blood was collected 1,3,7 and 14 days after ligation using pyrogen-free tubes (BD Biosciences, San Jose, CA, USA) and centrifuged at $3,000 \mathrm{rpm}$ for $20 \mathrm{~min}$ at $4^{\circ} \mathrm{C}$. The resulting serum was diluted 100 -fold with double-distilled water. Mouse tumor necrosis factor- $\alpha$ (TNF- $\alpha$ ) and interleukin- 6 (IL-6) were measured by ELISA according to the manufacturer's instructions (Boster, China), respectively. ELISA standards ranged from 15.6 to $1,000 \mathrm{pg} / \mathrm{ml}$. The absorbance was measured at $450 \mathrm{~nm}$.

Statistical analysis. Data were presented as the mean \pm standard deviation. Comparisons were made by the two-tailed Student's t-test for independent samples or one-way analysis of variance (ANOVA) and post hoc Scheffe's test as appropriate. $\mathrm{P}<0.05$ was considered statistically significant.

\section{Results}

Pam3CSK4 induced migration of HUVECs. The effect of Pam3CSK4 on the migration of HUVECs was assessed using wound repair assays. HUVECs migrated more rapidly in response to Pam3CSK4 stimulation after 18 h (Fig. 1), while 


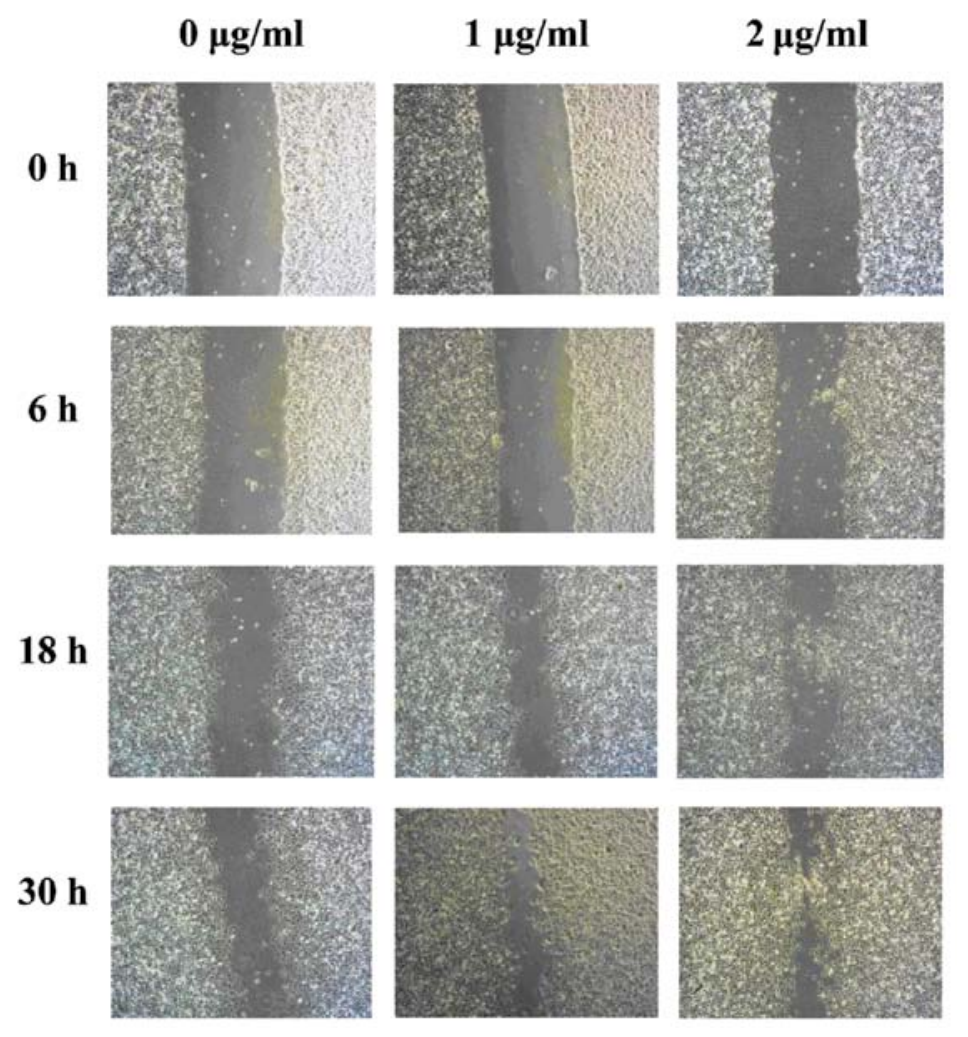

Figure 1. TLR-2 activation increased migration of HUVECs. No apparent difference was observed after $6 \mathrm{~h}$. HUVECs migrated more rapidly in response to Pam3CSK4 after 18 h, while after 30 h, HUVECs without Pam3CSK4 stimulation showed a clear wound. By contrast, Pam3CSK4 induced cell migration across the wound margins resulting in almost complete closure of the wound.
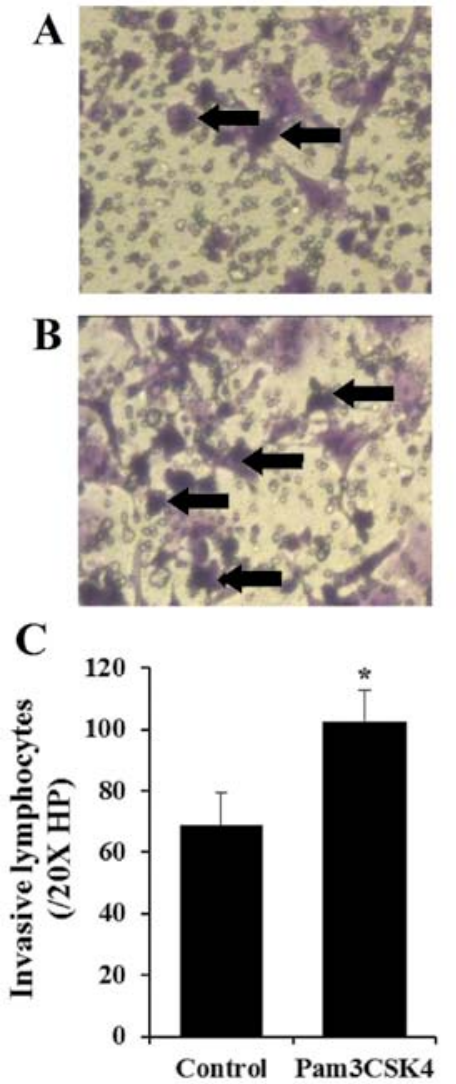

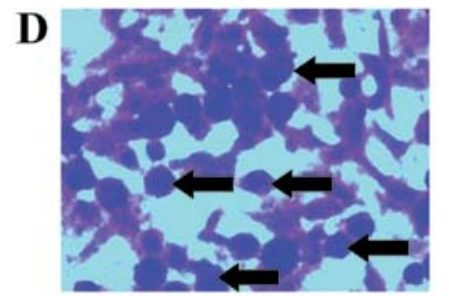

$\mathbf{E}$

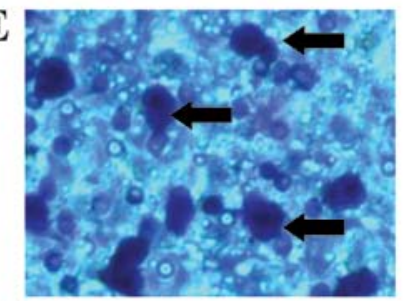

F

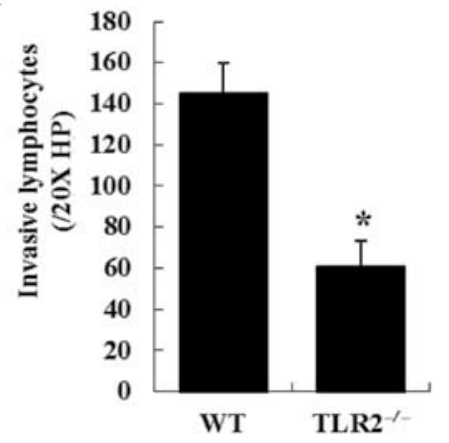

Figure 2. TLR2 activation increased the permeability of HUVECs and lymphocyte invasion. Representative photomicrographs show invasive lymphocytes. (A) Control group without pretreatment of HUVECs. (B) Transwell results with Pam3CSK4-pretreatment of HUVECs. (C) Bar graph quantifying invasive lymphocytes $(\mathrm{n}=4)$. " $\mathrm{P}<0.05$ compared to the value of the control. (D) Transwell results from WT mice. (E) Transwell results from TLR2 ${ }^{-/ 2}$ mice. (F) Bar graph quantifying invasive lymphocytes $(n=4)$. ${ }^{*} \mathrm{P}<0.05$ compared to the WT mice. 

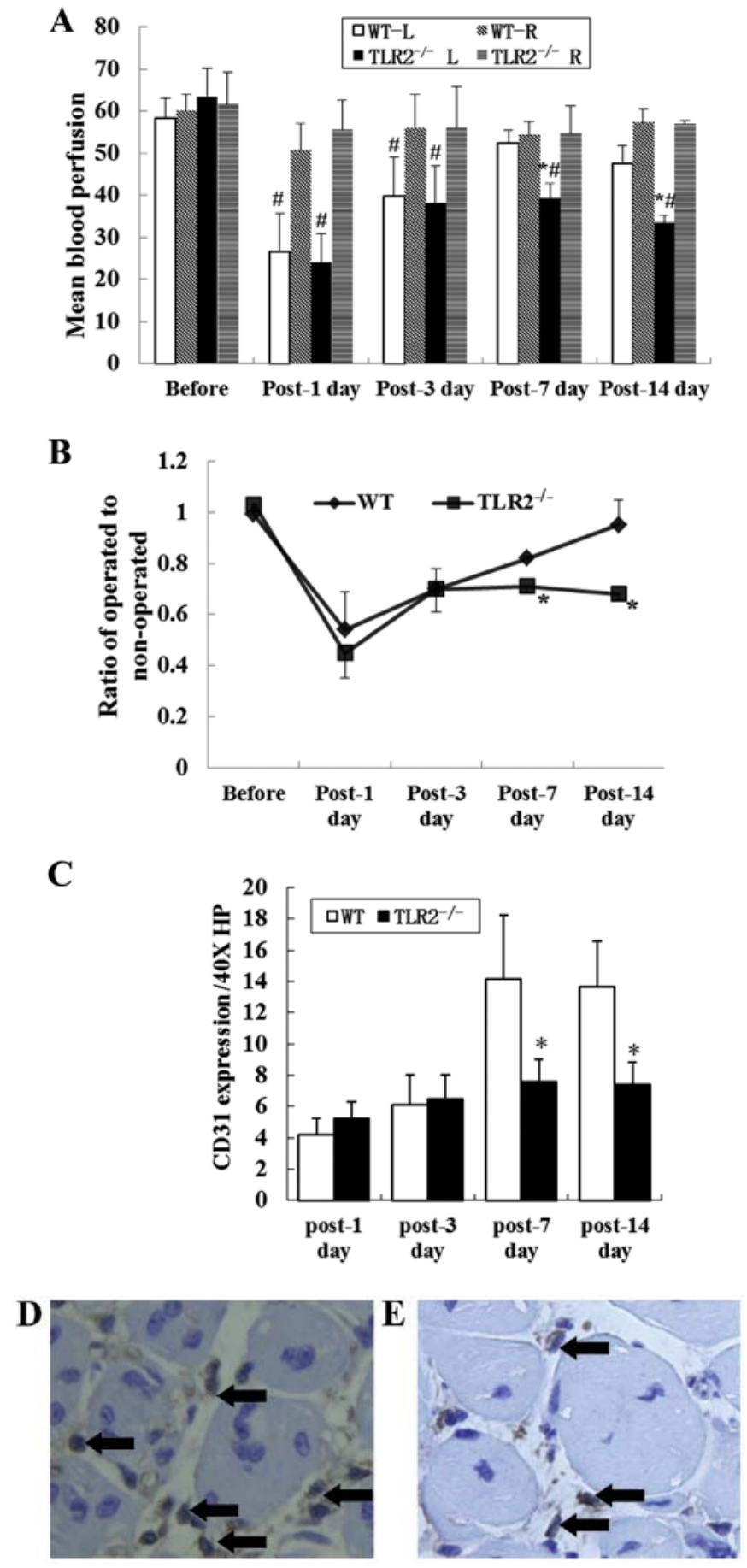

Figure 3. Blood perfusion and ischemic gastrocnemius CD31 expression in $\mathrm{WT}$ and TLR2 ${ }^{-/}$mice limbs. (A) L and R are the operated and non-operated limbs, respectively. Blood perfusion of operated limbs in TLR2 $2^{-}$mice was significantly lower than that in WT mice, on the 7th and 14th day after ligation. When compared to those of non-operated limbs, blood perfusion was significantly reduced in the ischemic limbs in the two groups on the 1st and 3rd day after ligation. On the 7th and 14th day after ligation, blood perfusion was still decreased in TLR $2^{--}$mice but no difference was observed in WT mice. ${ }^{~} \mathrm{P}<0.05$, TLR2 ${ }^{-/} \mathrm{L}$ vs. WT-L, ${ }^{\#} \mathrm{P}<0.05$, WT-L vs. WT-R, or TLR $2^{--} \mathrm{L}$ vs. TLR2 ${ }^{-/ R}$. (B) Blood perfusion ratio of operated to non-operated leg in each mouse reflected recovery of the ischemic limbs. The ratio in TLR2 ${ }^{-/}$mice was significantly lower than that in WT mice on the 7th and 14th day after ligation, but no significant difference was found on the 1st and 3rd day after ligation. " $\mathrm{P}<0.05$, TLR2 $2^{-/}$vs. WT (C) CD31 expression in ischemic gastrocnemiuses: TLR2 ${ }^{-\digamma}$ mice showed a reduced CD31 expression compared to WT mice on the 7th and 14th day after ligation. No significant difference was detected between the two groups of mice on the 1st and 3rd day after ligation. ${ }^{*} \mathrm{P}<0.05$, TLR $2^{-1}$ vs. WT mice. Representative photomicrograph shows the CD31 expression in (D) WT and (E) TLR $2^{-1-}$ mice. after 30 h, HUVECs without Pam3CSK4 stimulation showed a clear wound, where minimal cell migration across the wound margin was observed. By contrast, Pam3CSK4 induced cell migration across the wound margins resulting in almost complete closure of the wound.

Permeability of HUVECs and lymphocyte invasion. To assess the potential role of TLR2 activation in endothelial cell permeability, HUVECs were pretreated with Pam3CSK4 $(1 \mu \mathrm{g} / \mathrm{ml})$, and then seeded in Transwell membranes for lymphocyte invasion. Fig. 2A shows lymphocyte invasion under basal conditions compared to those of Pam3CSK4 pretreatment (Fig. 2B). Quantification of lymphocyte invasion was performed in six different fields/chamber under 20X high-power lens (HP), and the mean values were used to evaluate the invasive lymphocytes for each chamber. The lymphocytes were significantly increased by Pam3CSK4 pretreatment compared to the control $(102.29 \pm 10.60 / 20 X$ HP vs. $68.69 \pm 10.57 / 20 X$ HP, $\mathrm{P}<0.001)$ (Fig. 2C).

In the lymphocyte invasion assay, lymphocytes isolated from the peripheral blood of WT and TLR2 $2^{-/}$mice were placed in the upper well of the Transwell chamber seeded with HUVECs for lymphocyte migration. The lymphocytes invasion (Fig. 2D and E) was quantified in the same manner, and the results showed that the amount of invasive lymphocytes from the WT mice was significantly more than that from the TLR2 $2^{-/}$mice $(145.07 \pm 14.49 / 20 X$ HP vs. $60.92 \pm 12.27 / 20 X$ HP, $\mathrm{P}<0.001)$ (Fig. 2F).

$T L R 2^{-\%}$ delayed recovery from ischemic injury. Following hindlimb ligation surgery, the operated limb showed significant reduction in blood perfusion compared to the sham-operated limb. On the 1st and 3rd day after ligation, the operated limbs showed no differences in blood perfusion between TLR $2^{-/}$and WT mice ( $\mathrm{n}=6 /$ time-point/group, $24.05 \pm 6.69$ vs. $26.50 \pm 9.16$, $\mathrm{P}>0.05 ; 38.14 \pm 8.82$ vs. $39.61 \pm 9.45, \mathrm{P}>0.05)$. However, blood perfusion of operated limbs in TLR2 ${ }^{-/}$mice was significantly lower than that in WT mice on the 7th and 14th day after ligation ( $39.15 \pm 3.71$ vs. $52.40 \pm 2.93, \mathrm{P}=0.001 ; 33.47 \pm 1.69$ vs. 47.43 $\pm 4.27, \mathrm{P}=0.013$ ) (Fig. 3A).

To reduce the effect of individual diversities on data, the results were plotted as a ratio of operated to non-operated leg for each mouse, which reflected the recovery of ischemic leg. The recovered blood perfusion of operated limbs in TLR2 $2^{-1}$ mice was significantly lower than that in WT mice, on the 7th and 14th day after ligation, however, no difference was detected on the 1st and 3rd day after ligation (Fig. 3B).

TLR2 $2^{-}$inhibited angiogenesis following ischemic injury. To identify angiogenesis in the ischemic muscles, ischemic gastrocnemius muscles were collected on the 1st, 3rd, 7th and 14th day after ligation in order to detect $\mathrm{CD} 31^{+}$endothelial monolayer lining the lumen of vascular structures. TLR2 $2^{-1-}$ mice showed a reduced CD31 expression compared to WT mice on the 7th and 14th day after ligation ( $\mathrm{n}=6 /$ time-point/ group, $7.63 \pm 1.41 / 40 \mathrm{X}$ HP vs. $14.17 \pm 4.07 / 40 \mathrm{X} \mathrm{HP}, \mathrm{P}=0.001$; $7.44 \pm 1.42 / 40 \mathrm{X}$ HP vs. $13.67 \pm 2.92 / 40 \mathrm{X}$ HP, $\mathrm{P}<0.001)$. CD31 expression was also detected on the 1st and 3rd day after ligation, although there was no significant difference between the two groups of mice (Fig. 3C). 

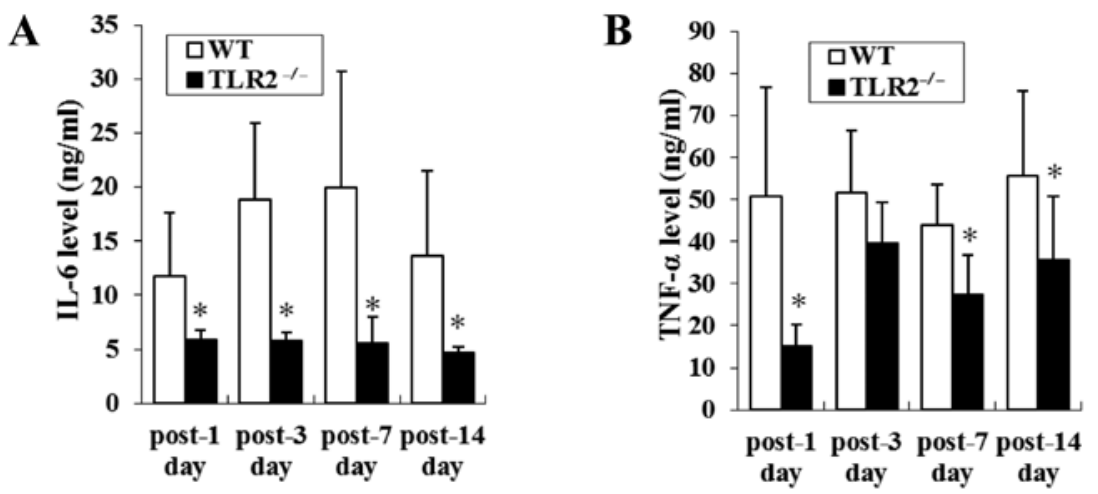

Figure 4. WT mice showed higher levels of serum IL-6 and TNF- $\alpha$ compared to TLR $2^{-/}$mice in ischemic injury. (A) Elevated levels of serum IL-6 were found at all time-points after surgery in WT mice, whereas little IL-6 was found in the serum of TLR2 ${ }^{-/}$mice. " P $<0.05$ TLR2 $2^{--}$vs. WT mice. (B) TNF- $\alpha$ levels in TLR2 ${ }^{-1-}$ mice were increased after surgery but were still significantly lower than those in WT mice on 1,7 , and 14 days after ligation. ${ }^{*} \mathrm{P}<0.05$ TLR2 ${ }^{-\digamma}$ vs. WT mice.

$T L R 2^{-\%}$ reduced $T N F-\alpha$ levels and IL- 6 release following ischemic injury. To examine the effect of TLR2 deficiency on cytokine production in ischemic injury, the sera of TLR2 $2^{-1-}$ and WT mice were collected to measure the levels of TNF- $\alpha$ and IL-6 1, 3, 7 and 14 days after ligation. Elevated levels of IL-6 were identified on 1, 3, 7 and 14 days after ligation in the serum of WT mice, whereas little IL- 6 was found in the serum of TLR2 ${ }^{-/}$mice $(11.79 \pm 5.80$ vs. $5.85 \pm 0.94 \mathrm{ng} / \mathrm{ml}, \mathrm{P}=0.043$; $18.91 \pm 7.03$ vs. $5.80 \pm 0.80 \mathrm{ng} / \mathrm{ml}, \mathrm{P}=0.021 ; 19.95 \pm 10.81$ vs. $5.60 \pm 2.39 \mathrm{ng} / \mathrm{ml}, \mathrm{P}=0.003 ; 13.68 \pm 7.80$ vs. $4.68 \pm 0.57 \mathrm{ng} / \mathrm{ml}$, $\mathrm{P}=0.002$ ) (Fig. 4A).

Similarly, TNF- $\alpha$ levels in TLR2 ${ }^{--}$mice were increased after ligation but were still significantly lower than those in WT mice on the 1st, 7 th and 14th day after ligation $(15.18 \pm 5.09$ vs. $50.80 \pm 25.97 \mathrm{ng} / \mathrm{ml}, \mathrm{P}=0.021 ; 27.35 \pm 9.44$ vs. $44.04 \pm 9.68 \mathrm{ng} / \mathrm{ml}$, $\mathrm{P}=0.005 ; 35.69 \pm 15.16$ vs. $55.74 \pm 20.08 \mathrm{ng} / \mathrm{ml}, \mathrm{P}=0.046)$ (Fig. $4 \mathrm{~B})$.

\section{Discussion}

Angiogenesis is an important mechanism that protects organs against imminent danger and tissue necrosis following acute ischemic injury in various diseases. Inflammatory signaling pathways are activated even in the absence of infection and are believed to play an important role in angiogenesis during wound healing. In the ischemic tissue, damage-associated molecular patterns (DAMPs), released from necrosis cells, activates TLR2 and sterile inflammation (17). The aim of this study, was to clarify whether the TLR2 signaling pathway induced angiogenesis to protect tissues that experienced ischemia.

Lymphocyte invasion and recruitment in the ischemic lesion are essential in inflammation and angiogenesis during ischemic injury healing. Endothelial cell adhesion, migration and permeability are also involved in this procedure. In this study, we demonstrated that TLR2 activation induced HUVEC cell migration, and increased its permeability to lymphocyte. TLR2 activation was able to promote tube formation, as well as endothelial cell invasion and migration in vitro (18). However, lymphocytes in peripheral blood are required to cross endothelial cells to assemble in ischemic tissue in vivo. Therefore, lymphocyte invasion and endothelial cell permeability are important, but have not been extensively studied. Thus, we isolated the lymphocytes from TLR2 $2^{-/}$and WT mice for invasion assay without Pam3CSK4 stimulation.
TLR2 ${ }^{-/}$lymphocytes showed a significantly reduced invasive ability compared to WT lymphocytes. The results suggest that the TLR2 signaling pathway was involved in lymphocyte invasion and endothelial cell permeability, promoting lymphocyte recruitment and serving as important factors of angiogenesis.

Having established the role of TLR2 in cell migration, invasion and endothelial cell permeability in vitro, we assessed the association between TLR2 and angiogenesis in vivo. An acute hindlimb ischemic model was produced, and all the mice showed hindlimb disability following ligation. However, $\mathrm{TLR}^{-/}$and WT mice showed different responses after ligation. On the 7th and 14th day after ligation, blood perfusion of ischemic legs in WT mice had almost recovered and showed no difference compared with non-operated legs. However, blood perfusion of ischemic legs in TLR $2^{--}$mice remained lower than that in non-operated and ischemic legs in WT mice. Our results suggest that TLR2 deficiency induced the delayed recovery of ischemic injury to leg muscles. Since the femoral artery was ligated and excised, the blood perfusion recovery resulted from neovascularization in ischemic muscles. To confirm our hypothesis, CD31 was used to evaluate the extent of revascularization. CD31, also known as platelet-endothelialcell adhesion molecule-1 (PECAM-1), is expressed at high density at lateral borders of endothelial cells (19-21), and is associated with angiogenesis. The revascularization labeled by CD31 in ischemic gastrocnemius muscles showed similar results with the blood perfusion results. The TLR2 $2^{-/}$mice also showed a significantly decreased expression of CD31 on the 7th and 14th day after ligation, but no difference was found on the 1st and 3rd day. These data suggested that the TLR2 signaling pathway is important in revascularization after ischemic injury, and promotes long-term recovery. After ischemic injury, inflammatory cytokine and proangiogenic factors were upregulated, and inflammatory cells including lymphocytes were recruited in ischemic tissue. In TLR $2^{--}$mice, the ability of cytokine recognition and arteriogenesis activation was apparently impaired, and the reduced blood flow eventually induced the delayed recovery of the ischemic legs. The importance of the TLR2 signaling pathway was not detected at the earlier period of ischemic injury, but was strongly verified in long-term revascularization.

A number of studies have clarified the relationship between inflammatory cytokines and angiogenesis (22-27). 
Additionally, TLR2 signaling contributed to the production of TNF- $\alpha$ and IL-6 (28-30). In the present study, we investigated whether TNF- $\alpha$ and IL- 6 were associated with differences in angiogenesis between TLR2 $2^{-/}$and WT mice. Expression of TNF- $\alpha$ and IL- 6 in TLR2 ${ }^{-/-}$mice was reduced in both the early and long-term period of ischemic injury. These findings suggest that the production of inflammatory cytokines in ischemic injury depend on TLR 2 expression in the host. After TLR2 ${ }^{-/-}$, the upregulated expression of NF- $\mathrm{KB}$ and AP-1 in the nucleus after zero-flow ischemia may apparently be reduced $(31,32)$, causing the transcription and expression of cytokine proteins (33) to be lower in TLR $2^{-/-}$mice compared to those of WT mice. In the earlier period of ischemic injury, levels of TNF- $\alpha$ and IL- 6 in WT mice were higher than those in TLR $2^{-/}$mice. However, the formation of new vessels was complicated and slow; therefore, detectable blood reperfusion and new vessels in ischemic legs showed no differences. The ischemic injury in WT mice had already recovered by the 7 th day after ligation. However, the neovascularization process in TLR $2^{-/-}$mice was significantly slower due to lack of cytokines. Furthermore, lymphocyte invasion was reduced in TLR $2^{-1-}$ mice. Our data suggest that TLR2 is important in recovery of the ischemic injury, and that it is closely associated with cytokine production and angiogenesis promotion of TLR2.

In conclusion, we have demonstrated that TLR2 activation promoted endothelial cell migration, cell permeability and lymphocyte invasion. TLR 2 activation promoted angiogenesis in vivo, which was connected to the serum of TNF- $\alpha$ and IL- 6 release, and lymphocyte invasion. These findings provide evidence that the TLR2 signaling pathway is potentially a new target for treating ischemic disease. Future studies should be performed to clarify the intracytoplasm mechanism.

\section{Acknowledgements}

This study was funded by the Science Technology Department of Zhejiang Province under grant no. 2012C33028.

\section{References}

1. Faxon DP, Fuster V, Libby P, et al: Atherosclerotic Vascular Disease Conference: Writing Group III: pathophysiology. Circulation 109: 2617-2625, 2004.

2. Dormandy J, Heeck L and Vig S: Acute limb ischemia. Semin Vasc Surg 12: 148-153, 1999.

3. Costantini V and Lenti M: Treatment of acute occlusion of peripheral arteries. Thromb Res 106: V285-V294, 2002.

4. Isner JM: Therapeutic angiogenesis: a new frontier for vascular therapy. Vasc Med 1: 79-87, 1996.

5. Chen YC, Hsiao CC, Chen CJ, et al: Toll-like receptor 2 gene polymorphisms, pulmonary tuberculosis, and natural killer cell counts. BMC Med Genet 11: 17, 2010.

6. Akira S, Uematsu S and Takeuchi O: Pathogen recognition and innate immunity. Cell 124: 783-801, 2006.

7. Medzhitov R, Preston-Hurlburt P and Janeway CA Jr: A human homologue of the Drosophila Toll protein signals activation of adaptive immunity. Nature 388: 394-397, 1997.

8. Carmeliet P: Angiogenesis in life, disease and medicine. Nature 438: 932-936, 2005.

9. Shigeoka AA, Holscher TD, King AJ, et al: TLR2 is constitutively expressed within the kidney and participates in ischemic renal injury through both MyD88-dependent and -independent pathways. J Immunol 178: 6252-6258, 2007.

10. Leemans JC, Stokman G, Claessen N, et al: Renal-associated TLR2 mediates ischemia/reperfusion injury in the kidney. J Clin Invest 115: 2894-2903, 2005.
11. Favre J, Musette P, Douin-Echinard V, et al: Toll-like receptors 2-deficient mice are protected against postischemic coronary endothelial dysfunction. Arterioscler Thromb Vasc Biol 27: 1064-1071, 2007.

12. Mersmann J, Berkels R, Zacharowski P, et al: Preconditioning by Toll-like receptor 2 agonist Pam3CSK4 reduces CXCL1dependent leukocyte recruitment in murine myocardial ischemia/ reperfusion injury. Crit Care Med 38: 903-909, 2010.

13. Hua F, Ma J, Ha T, et al: Preconditioning with a TLR2 specific ligand increases resistance to cerebral ischemia/reperfusion injury. J Neuroimmunol 199: 75-82, 2008.

14. Ha T, Lu C, Liu L, et al: TLR2 ligands attenuate cardiac dysfunction in polymicrobial sepsis via a phosphoinositide 3-kinase-dependent mechanism. Am J Physiol Heart Circ Physiol 298: H984-H991, 2010.

15. Chang YJ, Wu MS, Lin JT and Chen CC: Helicobacter pyloriinduced invasion and angiogenesis of gastric cells is mediated by cyclooxygenase-2 induction through TLR2/TLR9 and promoter regulation. J Immunol 175: 8242-8252, 2005.

16. Scholz D, Ziegelhoeffer T, Helisch A, et al: Contribution of arteriogenesis and angiogenesis to postocclusive hindlimb perfusion in mice. J Mol Cell Cardiol 34: 775-787, 2002.

17. Martin P, D'Souza D, Martin J, et al: Wound healing in the PU.1 null mouse - tissue repair is not dependent on inflammatory cells. Curr Biol 13: 1122-1128, 2003.

18. Saber T, Veale DJ, Balogh E, et al: Toll-like receptor 2 induced angiogenesis and invasion is mediated through the Tie 2 signalling pathway in rheumatoid arthritis. PLoS One 6: e23540, 2011.

19. Muller WA, Ratti CM, McDonnell SL and Cohn ZA: A human endothelial cell-restricted, externally disposed plasmalemmal protein enriched in intercellular junctions. J Exp Med 170: 399-414, 1989.

20. Newman PJ, Berndt MC, Gorski J, et al: PECAM-1 (CD31) cloning and relation to adhesion molecules of the immunoglobulin gene superfamily. Science 247: 1219-1222, 1990.

21. Muller WA: Leukocyte-endothelial-cell interactions in leukocyte transmigration and the inflammatory response. Trends Immunol 24: 327-334, 2003.

22. Balkwill F: Tumour necrosis factor and cancer. Nat Rev Cancer 9: 361-371, 2009

23. Balkwill F: TNF-alpha in promotion and progression of cancer. Cancer Metastasis Rev 25: 409-416, 2006.

24. Lin WW and Karin M: A cytokine-mediated link between innate immunity, inflammation, and cancer. J Clin Invest 117: 1175-1183, 2007.

25. Ancrile B, Lim KH and Counter CM: Oncogenic Ras-induced secretion of IL6 is required for tumorigenesis. Genes Dev 21: 1714-1719, 2007.

26. Huang SP, Wu MS, Shun CT, et al: Interleukin-6 increases vascular endothelial growth factor and angiogenesis in gastric carcinoma. J Biomed Sci 11: 517-527, 2004.

27. Fan Y, Ye J, Shen F, et al: Interleukin-6 stimulates circulating blood-derived endothelial progenitor cell angiogenesis in vitro. J Cereb Blood Flow Metab 28: 90-98, 2008.

28. Lin H, Yan J, Wang Z, et al: Loss of immunity-supported senescence enhances susceptibility to hepatocellular carcinogenesis and progression in TLR2-deficient mouse. Hepatology: Aug 1, 2012 (Epub ahead of print). doi: 10.1002/hep.25991.

29. Amura CR, Renner B, Lyubchenko T, Faubel S, Simonian PL and Thurman JM: Complement activation and Toll-like receptor-2 signaling contribute to cytokine production after renal ischemia/ reperfusion. Mol Immunol 52: 249-257, 2012.

30. Correa-Costa M, Braga TT, Semedo P, et al: Pivotal role of Toll-like receptors 2 and 4, its adaptor molecule MyD88, and inflammasome complex in experimental tubule-interstitial nephritis. PLoS One 6: e29004, 2011.

31. Xie W, Wang Y, Huang Y, Yang H, Wang J and Hu Z: Toll-like receptor 2 mediates invasion via activating NF-kappaB in MDA-MB-231 breast cancer cells. Biochem Biophys Res Commun 379: 1027-1032, 2009.

32. Shuto T, Ono T, Ohira Y, et al: Curcumin decreases Toll-like receptor-2 gene expression and function in human monocytes and neutrophils. Biochem Biophys Res Commun 398: 647-652, 2010.

33. Jang JH, Yang ES, Min KJ and Kwon TK: Inhibitory effect of butein on tumor necrosis factor- $\alpha$-induced expression of cell adhesion molecules in human lung epithelial cells via inhibition of reactive oxygen species generation, $\mathrm{NF}-\mathrm{\kappa B}$ activation and Akt phosphorylation. Int J Mol Med 30: 1357-1364, 2012. 